in rearrangement of the clathrin lattice, and/or may function to signal further steps in the process. Surprisingly, this read of the data would provide no evidence for the formation near the substrate of a substantial dynamin collar around a CP neck, as the dynamin TIRF signal has declined virtually to background before significant invagination of the lattice has occurred. Finally, it is striking that actin is recruited only after the $\mathrm{CP}$ is substantially invaginated and that the corresponding TIRF signal decreases with that of clathrin. This may suggest that both proteins recede in tandem from the plane of the PM, implicating actin in an intimate role in the birth of a coated vesicle. With the sophisticated work of Merrifield et al., we can almost see the CP — a specialized collection device in communication with the environment - transforming into an internalized transport vesicle.

Francesca Santini and James H. Keen are in the Deptartment of Microbiology and Immunology, Cellular Biology and Signaling Program, Kimmel Cancer Center, Thomas Jefferson University,

Philadelphia, PA 19107, USA

e-mail:Jim.Keen@mail.tju.edu

1. Lafer, E. M. Traffic 3, 513-520 (2002).

2. Thompson, H. M. \& McNiven, M. A. Curr. Biol. 11, R850

(2001)

3. Qualmann, B., Kessels, M. M. \& Kelly, R. B. J. Cell Biol. 150,
F111-F116 (2000).

4. Gaidarov, I., Santini, F., Warren, R. A. \& Keen, J. H. Nature Cell Biol. 1, 1-7 (1999).

5. Merrifield, C. J., Feldman, M. E., Wan, L. \& Almers, W. Nature Cell Biol. 4, 691-698 (2002).

6. Wu, X. et al. J. Cell Biol. 155, 291-300 (2001).

7. Poodry, C. A. \& Edgar, L. J. Cell Biol. 81, 520-527 (1979).

8. van der Bliek, A. M. \& Meyerowitz, E. M. Nature 351, 411-414 (1991).

9. Damke, H., Baba, T., Warnock, D. E. \& Schmid, S. L. J. Cell Biol. 127, 915-934 (1994).

10. Tsuboi, T. et al. J. Biol. Chem. 277, 15957-15961 (2002).

11. Kubler, E. \& Riezman, H. EMBO J. 12, 2855-2862 (1993).

12. Fujimoto, L. M., Roth, R., Heuser, J. E. \& Schmid, S. L. Traffic 1, 161-171 (2000).

13. Santini, F., Gaidarov, I. \& Keen, J. H. J. Cell Biol. 156, 665-676 (2002).

14. Baba, T., Ueda, H., Terada, N., Fujii, Y. \& Ohno, S. J. Histochem. Cytochem. 47, 637-648 (1999).

\title{
First mitotic division: getting it right at the start
}

At fertilization, the mouse oocyte completes the second meiotic division and cleaves asymmetrically into a large daughter oocyte and a small second polar body (PB). By convention, the $\mathrm{PB}$ marks the animal pole of the zygote and the diametrically opposite side is, by default, the vegetal pole. The plane of first cleavage of the zygote passes through these two poles, as well as the sperm entry point (SEP) on the surface of the oocyte. The orientation of this plane impacts significantly on the lineage differentiation of the blastomeres of the 2-cell embryo and the allocation of their descendants to the embryonic and abembryonic compartments of the blastocyst (see Figure).

A study by Plusa et al. (Nature Cell Biol. 4, 811-815 (2002)) shows that the ablation of the animal pole, but not the vegetal pole, causes misalignment of the first cleavage plane so that it no longer passes through the vegetal pole regularly. However, removing either the animal or the vegetal pole portion of the zygote does not affect its ability to form a blastocyst. A more marked disruption of first cleavage of the zygote is observed when an extra fragment of the whole animal pole, or only the cytoplasmic fragment containing the remnant of the second meiotic spindle (the mid-body), is added to the zygote. The cleavage plane aligns randomly with one or both animal poles and cell division is accompanied by aberrant segregation of chromosomes during mitosis, reluctance of the spindle to move towards the meiotic mid-body during cytokinesis and consequently arrest of embryonic development. In contrast, the presence of extra vegetal pole material does not cause any deleterious effect. Furthermore, a more severe interference with the cleavage plane is found when the extra animal pole material is located closer to the pre-existing animal pole. These findings suggest that some undefined factors in the animal pole cytoplasm are critical for the orientation of the cleavage plane and that they may function in a dose- and site-dependent manner.

This new work highlights a previously unrecognized role of the animal pole in determining the plane of first cleavage. The attachment of mitotic spindle ends through microtubules to the animal pole, where the meiotic midbody is localized, implies that the spindle may be oriented by a dynamic process of capture and release of the spindle by cytoplasmic materials from previous rounds of cytokinesis. However, fixation of the spindle to the

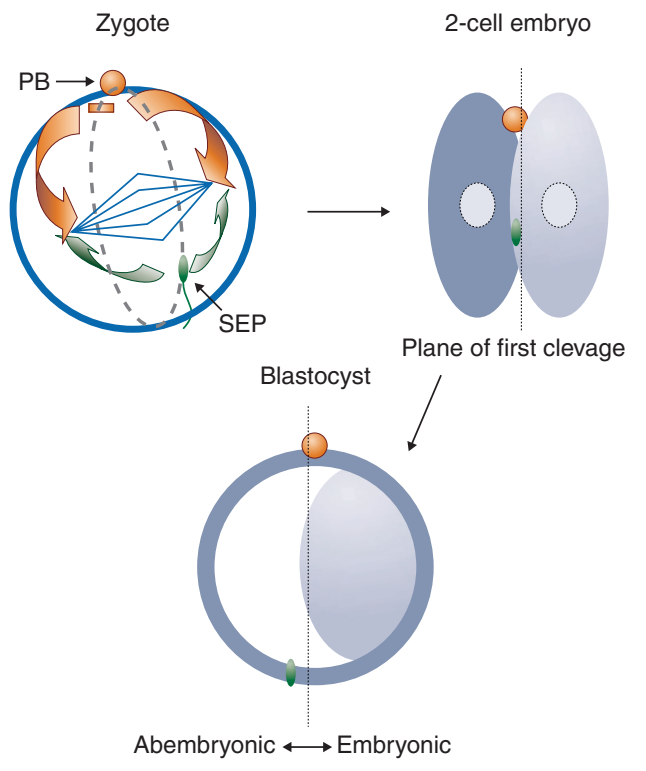

animal pole can only position the spindle relative to the animal-vegetal axis. An additional anchorage activity is required to rotate the spindle in the equatorial plane, such that cleavage plane is aligned with the meridian defined by the position of the SEP (see Figure). Further elucidation of the cascade of molecular events triggered by the extrusion of the $\mathrm{PB}$ and sperm entry will reveal the effects of polarization of the cytoplasm, distribution of proteins and re-arrangement of the cytoskeleton on spindle orientation.

PATRICK P. L. TAM

Children's Medical Research Institute, University of Sydney, Australia e-mail:ptam@cmri.usyd.edu.au 